\title{
EXPEDIENTE
}

\section{EDITOR CIENTÍFICO}

Celia Linhares, UFRRJ/PPGEDUC, Brasil

\section{EDITORES GERENTES}

Adrianne Ogêda Guedes, UNIRIO, Brasil

Márcia Denise Pletsch, UFRRJ - PPGEDUC, Brasil

Rosimeri de Oliveira Dias, FFP/UERJ, Brasil

\section{COMISSÃO EDITORIAL}

Adrianne Ogêda Guedes, UNIRIO, Brasil

Denize Sepúlveda, FFP/UERJ, Brasil

Jonas Alves da Silva Jr., UFRRJ/IM, Brasil

Léa Velocina Vargas Tiriba, UNIRIO, Brasil

Marcia Denise Pletsch, UFRRJ/IM, Brasil

Rosimeri de Oliveira Dias, FFP/UERJ, Brasil

Vania Finholdt Leite, FFP/UERJ, Brasil

\section{EDITORAS DOSSIÊ ITINERÂNCIAS ENTRE MICHEL FOUCAULT E EDUCAÇÃO}

Rosimeri de Oliveira Dias, UERJ, Brasil

Denize Sepulveda, UERJ, Brasil

Vânia Leite, UERJ, Brasil

\section{ORGANIZAÇÃO DO DOSSIÊ}

Rosimeri de Oliveira Dias, FFP/UERJ, Brasil

\section{CONSELHO EDITORIAL}

Prof $^{a}$ Dr $^{a}$ Ana Maria Monteiro, UFRJ, Brasil

Prof. Dr. Carlos Eduardo Ferraço, Professor Associado da UFES, Brasil

Prof. Dr. Carlos Skliar, FLACSO-Argentina, Brasil

Prof $^{a} \operatorname{Dr}^{a}$ Catherine Walsh, Profesora de la Universidad Andina Simón Bolivar/Equador, Equador

Prof $^{a}$ Dr $^{a}$ Filomena Maria de Arruda Monteiro, Professora Associada da UFMT, Brasil

Prof. Dr. Guilherme do Val Toledo Prado, UNICAMP, Brasil

Prof $^{a}$ Dr $^{a}$ Inês Assunção de Castro Teixeira, Professora Associada da UFMG, Brasil 
Prof $^{a}$ Dr $^{a}$ Maura Corcini Lopes

Prof $^{a}$ Dr $^{\mathrm{a}}$ Maria Teresa Esteban, Professora Associada da UFF, Brasil

Prof. Dr. Marcos Reigotta, Professor Titular da UNISO, Brasil

Prof Nelson De Luca Pretto, Professor Titular da Faculdade de Educação da Universidade Federal da

Bahia, Brasil

Prof $^{a}$ Dr $^{a}$ Nilma Lino Gomes, Professora Adjunta da UFMG, Brasil

Prof $^{a}$ Dr $^{a}$ Virginia de Oliveira Silva, Professora Adjunto da UFPB, Brasil

\section{REVISORES}

Carlos César de Oliveira

Eurídice Hespanhol Macedo Pessoa

Fabiana Rodrigues

Fernanda Cavalcanti de Mello

Helenice Lopes Ribeiro Gomes

Liliana Secron Pinto

Maíra Marins da Silva

\section{EDITORES DE APOIO}

Carlos César de Oliveira

Izadora Martins da Silva de Souza

Letícia Pacheco de Mello Trotte

CAPA

Diana Dias, Brasil

BOLSISTA DE EXTENSÃO UERJ

Fabiana de Mesquita do P Dias, FFP/UERJ, Brasil 